\title{
Tragwerkslehre
}

Skelettbau und Wandbau

Von Professor Dipl.-Ing. Werner Herget Fachhochschule Darmstadt 
Die Deutsche Bibliothek - CIP-Einheitsaufnahme

\section{Herget, Werner:}

Tragwerkslehre: Skelettbau und Wandbau / von Werner Herget.

- Stuttgart: Teubner, 1993

ISBN-13: 978-3-519-05245-6

DOI: $10.1007 / 978-3-322-84844-4$

Das Werk einschließlich aller seiner Teile ist urheberrechtlich geschützt. Jede Verwertung außerhalb der engen Grenzen des Urheberrechtsgesetzes ist ohne Zustimmung des Verlages unzulässig und strafbar. Das gilt besonders für Vervielfältigungen, Übersetzungen, Mikroverfilmungen und die Einspeicherung und Verarbeitung in elektronischen Systemen.

(c) B. G. Teubner Stuttgart 1993

Umschlaggestaltung: Peter Pfitz, Stuttgart 


\section{VORWORT}

Tragen ist ein strategisches Problem bei Kräften, die in einem Bauwerk entstehen und nicht in ihrer Wirkungslinie in den Baugrund abgeleitet werden können, ebenso ein ökonomisches bei der materialgerechten Dimensionierung der Bauteile an die abzutragenden Kräfte.

Die Lösung der ersten Teilaufgabe führt zum Tragwerk, einem Zusammenschluß aller tragenden Elemente des Bauwerkes, das eine kontinuierliche und den Formvorstellungen des Planers adäquate Kraftableitung gewährleistet. Die Tragwerkslehre bietet Konzepte auf der Basis eines Zusammenhangsverständnisses, das den Architekten zur Tragwerksplanung ohne mathematische Algorithmen befähigt.

Das zweite Problem wird durch die Statik gelöst, zu einem Zeitpunkt im Planungsprozeß, zu dem die qualitativen Ergebnisse des ersten Teils bereits vorliegen. Daher ist Tragwerkslehre weder Statik, noch kann sie diese ersetzen.

Tragwerkslehre existiert als Lehrfach an den Architekturfachbereichen der Fachhochschulen. Da es in der Regel von Statikern vertreten wird, orientieren sich seine Inhalte überwiegend an einer "Statik für Architekten", weniger an den Zusammenhangsproblemen des Tragwerkes, in keinem Fall jedoch an einer systematischen Darstellung dieser Thematik. In Kreisen der Architekturdozenten besteht weitgehend Einvernehmen über notwendige inhaltliche Veränderungen, die jedoch außerordentlich lange Zeit in Anspruch nehmen.

Diese Lücke will das vorliegende Buch füllen.

Seine Lehrinhalte behandeln Skelett- und Wandbauwerke, die aus stabförmigen Elementen, Platten und Scheiben zusammengesetzt sind und bei Hochbauten das überwiegende Potential repräsentieren. Die Typenvielfalt der Skelette wird katalogisiert, - bei eingeschoßigen nach dem Stützungsumfang in der Haupttragebene, bei mehrgeschoßigen nach den Konstruktionsbaustoffen - ihr Aufbau analysiert und die Grundprinzipien variiert. An Literaturbeispielen werden Synthesepraxis und das Wechselspiel Form - Konstruktion dokumentiert.

Bei den Wandbauweisen in Mauerwerk und Stahlbeton entfällt das Problem einer besonderen Erzeugung des Tragwerkes, da dies in den Elementen Platte, Scheibe und Ringanker bereits latent enthalten ist. Die Lehrinhalte erstrecken sich daher auf Aufbau und Nachweis der Funktionsfähigkeit dieser Elemente und ihrer Verbindungen.

Diesen Teilen vorgeschaltet sind Übersichten über Lasten und Kräfte an Bauwerken, sowie über die Funktionsmechanismen der Tragelemente, soweit dies für das Tragwerksverständnis notwendig erschien. 
Nachgeschaltet sind Planungshilfen, velche die Entscheidungsfindung für oder gegen eine Tragwerksform in konstruktiver, baubetrieblicher und wirtschaftlicher Hinsicht erleichtern sollen, sowie Hinweise auf Entwurfshilfen in der Literatur.

Das Buch ist umfangreich mit Zeichnungen illustriert. Dies hat unterschiedliche Gründe: nach wie vor ist die Zeichnung Sprache des Architekten und Ingenieurs. Die der Statik entlehnten Strichsysteme ermöglichen mit wenig Aufwand hohe Informationsdichten und unterstützen außerdem die vornehmlich visuelle Aufnahmefähigkeit der Architekturstudenten. Die hohe Zeichnungsdichte erlaubt eine Reduzierung des Textumfanges. Das damit entstehende didaktische Problem, Text und Bilder zu harmonisieren, mußte im Layout gelöst werden.

Für diese im üblichen Lehrbuchaufbau ungewohnte Konstellation hat der Verlag sich sehr entgegenkommend und experimentierfreudig erwiesen. Dafür, wie für die Annahme des Projektes, das Bestehendes durch Neues erweitern und ersetzen will, gilt dem Verlag mein Dank.

Dank gebührt auch meiner Frau Gaby für die Reinschrift, die Erstellung der Zeichnungen und die Seitengestaltung.

Das Buch wendet sich an Fachhochschulstudenten der Architektur und Innenarchitektur (wegen des angestrebten eingeschränkten Bauvorlagerechtes). Dem Studenten des konstruktiven Ingenieurbaues wird es empfohlen, weil die Lehrerfahrung zeigt, daß selbst umfangreiche statische Kenntnisse weder das notwendige Zusammenhangsverständnis garantieren, noch die Betrachtungsweise des Tragwerkes als form- und raumbildendes Element ermöglichen.

Mir bleibt die Hoffnung auf eine positive Aufnahme der mitgeteilten Inhalte durch Studenten und Kollegen, wie auf deren Einarbeitung in das Lehrfach. Für kritische Anmerkungen bin ich dankbar. 


\section{BAUWERK UND TRAGWERK}

1.1 Die drei Entwurfskomponenten der Bauaufgabe ................. 1

1.2 Die Rolle des Tragwerkes im Entwurf ........................... 3

1.3 Das Beispiel der alten Baumeister ................................ 5

1.4 Tragwerkskenntnisse - Wieviel davon braucht der Architekt? 15

\section{LASTEN UND KRÄFTE}

2.1 Übersicht ......................................................... 19

2.2 Ständige Lasten ................................................. 20

2.3 Verkehrslasten.................................................... 26

2.3.1 Verkehrslasten aus Nutzung ............................. 26

2.3.2 Umweltbedingte Lasten ................................... 28

2.4 Trägheitskräfte .................................................... 32

2.5 Zwängungskräfte ................................................ 33

2.6 Fugen............................................................ 34

2.7 Überlagerung von Lasten und Kräften ......................... 37

\section{GRUNDLAGEN DES TRAGENS}

3.1. Anforderungen ................................................ 39

3.2 Unterschiede in den Bauweisen ................................. 41

3.3 Tragelemente .......................................................... 44

3.3.1 Fundamente ............................................... 44

3.3.2 Stützen ................................................. 49

3.3.3 Vollwandige Biegeträger .................................. 55

3.3.4 Fachwerkträger .......................................... 68

3.3.5 Rahmen................................................ 73

\section{SKELETTBAU}

4.1 Definition ............................................................. 75

4.2 Typologie.......................................................... 79

4.2.1 Bezeichnungen........................................... 79

4.2.2 Systeme der Nebenträger................................. 80

4.2.3 Haupttragsysteme......................................... 86

4.3. Freistehende Kragträger ....................................... 95

4.3.1 Grundsystem ........................................... 95

4.3.2 Variantenbildung zum Grundsystem ...................... 105

4.3.3 Objektbeispiele I - X.................................... 114 
4.4 Eingeschoßige Tragwerke auf 2 Stützen

(einschiffige Hallen) ................................................ 132

4.4.1 Abstützung / Abspannung ............................. 132

4.4.2 Fußeingespannte Stützen................................. 143

4.4.3 Biegesteife Ecken (Rahmen) ............................ 146

4.4.4 Dreigelenkstabzug, Bogen ............................... 155

4.4.5 Radiale Anordnung der Hallentragwerke .................. 158

4.4.6 Objektbeispiele XI - XIX ............................... 159

4.5 Eingeschoßige mehrschiffige Hallen ........................... 173

4.6 Mehrgeschoßige Skelette ...................................... 177

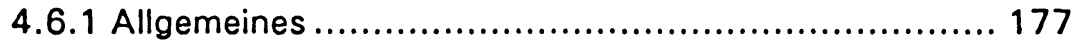

4.6.2 Zweigeschoßige Holzskelette.............................. 179

4.6.3 Stahlskelette mit Objektbeispielen XX, XXI............ 181

4.6.4 Stahlbetonskelette ........................................ 197

5 WANDBAU

5.1 Kriterien............................................................. 206

5.2 Verformungsverhalten der Wände.............................. 209

5.3 Die Funktionen der Schachtel..................................... 212

5.4 Die Bauweisen................................................... 214

5.5 Die Bauarten ................................................... 220

5.5.1 Mauerwerksbau.......................................... 220

5.5.2 Stahlbetontafelbau........................................ 225

5.5 .3 Holztafelbau.............................................. 227

6 ENTSCHEIDUNGS- UND ENTWURFSHILFEN ZUR TRAGWERKSPLANUNG

6.1 Der Planungsprozeß.......................................... 229

6.2 Entscheidungshilfen I (Vorplanung) ............................. 231

6.3 Entscheidungshilfen II (Entwurfsplanung)....................... 235

6.4 Entwurfshilfen ............................................ 242

LITERATURVERZEICHNIS .......................................... 253

STICHWORTVERZEICHNIS ........................................ 255 